\title{
ASOCIACIONES PALINOLOGICAS DE LAS FORMACIONES COLON Y MOLINO NOR-ORIENTE COLOMBIANO ${ }^{2}$
}

\author{
Hernando Dueñas Jimenez ${ }^{1}$
}

RESUMEN

Se describen las asociaciones palinológicas de muestras provenientes del tope de la Formación Colón (Cuenca del Catatumbo) y del tope de la Formación Molino (Cuenca del Cesár). Estas asociaciones se caracterizan por presentar como elementos principales Buttinia andreevi, Proteacidites dehaani, Spinizonocolpites baculatus, Spinizonocolpites echinatus, Echitriporites trianguliformis y Araucariacites sp., acompañados de Palaeocystodinium sp., Ceratiopsis sp. y Dinogymium acuminatum entre otros palinomorfos.

Estas asociaciones palinologicas permiten datar los topes de las Formaciones Colón y Molino como Maastrichtianas (Zona de Proteacidites dehaani/Palaeocystodinium sp.).

Palinológicamente las Formaciones Molino y Colón pueden ser correlacionadas con la Formación Umir en el Valle Medio del Magdalena y con la Formación Guaduas (parte inferior) de la Cuenca del Valle Superior del Magdalena.

\section{ABSTRACT}

The palynological associations of samples from the upper parts of the Colon Formation (Catatumbo Basin) and the Molino Formation (Cesar Basin) are described here. These associations are characterized by the following principal elements: Buttinia andreevi, Proteacidites dehaani, Spinizonocolpites baculatus, Spinizonocolpites echinatus, Echitriporites trianguliformis and Araucariacites sp., along with Palaeocystodinium sp., Ceratiopsis sp. and Dinogymium acuminatum amongst other palynomorphs.

These palynological associatons enable one to date the top of the Colón and Molino Formations as Maastrichtian (Zone: Proteacidites dehaani/Palaeocystodinium sp.).

The Molino and Colón Formations can be correlated palynologically with the Umir Formation in the Middle Valley of Magdalena and with the Guaduas Formation (lower part) of the Upper Valley of the Magdalena Basin.

\section{INTRODUCCION}

El presente trabajo describe las asociaciones palinológicas encontradas en muestras provenientes de la Formación Colón (Cuenca del Catatumbo) y de la Formación Molino (Cuenca del Cesár). Estas formaciones se caracterizan por ser predominantemente arcillosas y presentar pequeñas intercalaciones de arenas y calizas.

El ambiente de deposito de éstas formaciones varia de nerítico inferior en la base a lagunar marino hacia el tope, en donde se presentan arcillas carbonosas que han arrojado buenas asociaciones palinologicas las cuales han permitido asignar a éstos sedimetos una edad Maastrichtiana.

1 BIOSS Ltda. A.A. 52514 , D.E. Colombia.

2 Contribución a los proyectos 237 e 242. IUGS/UNESCO. 
FORMACION COLON

La Cuenca del Catatumbo se encuentra localizada al Nor-Oriente del territorio de Colombia, con un área aproximada de 7.000 kilómetros cuadrados (Fig. 1).

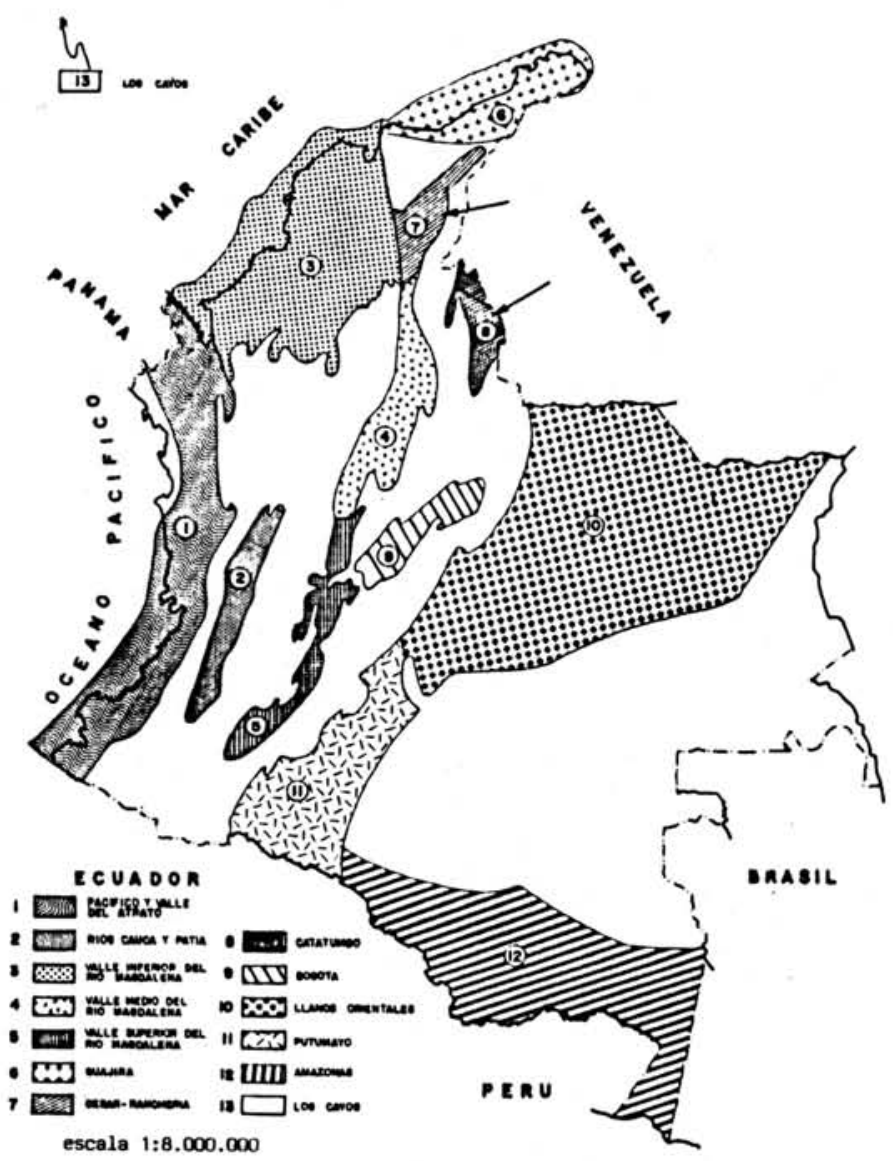

Fig. 1 - Cuencas sedimentarias de Colombia.

El potencial petrolero de ésta Cuenca se estableció desde 1920 y en la actualidad, con sus ocho campos petroleros presenta una producción acumulada (Dic. 1985) de $424.0 \mathrm{MmBls}$ de petróleo y 490.0 MMMc de gas.

Sedimentos del Aptiano al Reciente conforman la columna estrátigrafica de ésta Cuenca (Fig. 2). La Formación Tibú (Aptiano) descansa inconforme sobre basamento ígneo. Se presenta también dos inconformidades regionales en el Eoceno Inferior - Eoceno Medio y en el Mioceno Medio.

Desde el punto de vista petrolero, ésta es una Cuenca de objetivos múltiples en el Cretáceo. La mayor parte de las Unidades Cretácicas son productoras (Grupo Uribante, Formación Cogollo, Mito Juan) y algunas de ellas son rocas generadoras de aceite (Formación La Luna, Formación Colón).

El petróleo producido de las Unidades Cretácicas presenta un rango en su gravedad específica (API) de 25 a 54 grados. Producción de petróleo y gas también se obtiene de las Formaciones Terciarias (Carbonera, Mirador, Barco, Catatumbo) las cuales presentan un aceite de 17 a 37 grados API. 
Bol. IG-USP, Publ.Esp., 7:173-181, 1989

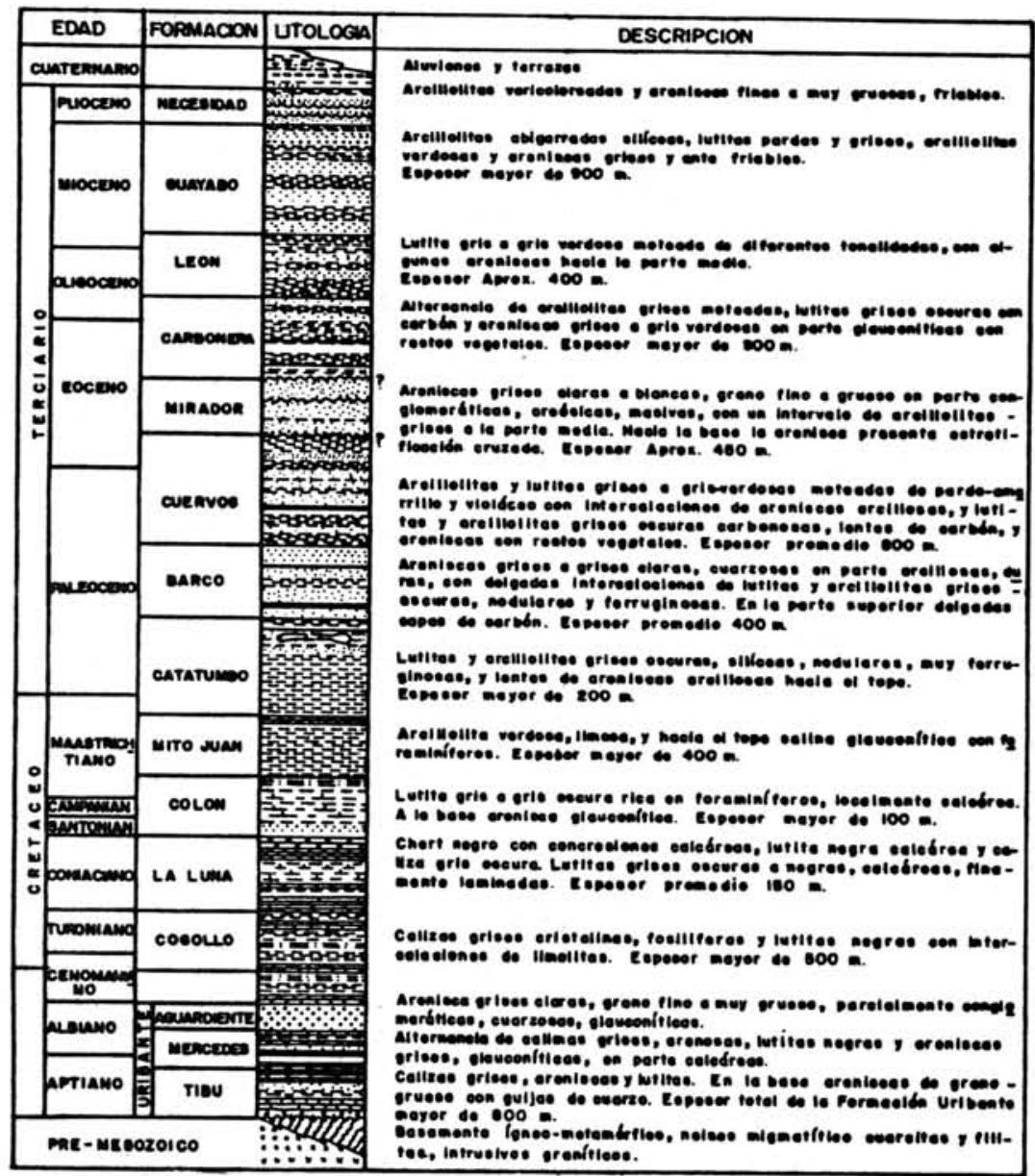

Fig. 2 - Cuenca del Catatumbo - columna estratigráfica generalizada.

La Formacion Colón también es conocida como Colón Shale. Es una sequencia predominantemente arcillosa en la cual se intercalan arcillas gris claras, gris oscuras, negras y pequeños lentes de arenas y calizas. Con base en foraminíferos, a ésta secuencia marina se le ha asignado una edad del Santoniano al Maastrichtiano.

En la base de la Formación Colón se presenta una arena glauconítica que recibe el nombre del Miembro Tres Esquinas, el cual reposa conformablemente sobre las calizas de la Formación La Luna.

La parte inferior de la Formación Colón presenta un buen recobro de foraminíferos dentro de los cuales se destaca la presencia de Siphogenerinoides bramlettei y Siphogenerinoides cretacea lo cual permite asignar a éstos sedimentos una edad Campaniano - Maastrichtiano. La parte más superior de la formación se caracteriza por la presencia de Ammobaculites columbianus, lo cual es base para asignarle una edad Maastrichtiana Tardia.

El ambiente de depósito de la Formación Colón es predominantemente marino, variando de nerítico inferior en la base a lagunar marino hacia el tope.

Muestras colectadas en el tope de la Formación Colón arrojaron muy buenas asociaciones palinológicas caracterizadas por la presencia de los siguientes palinomorfos (Plancha 1): 
POLEN Y ESPORAS

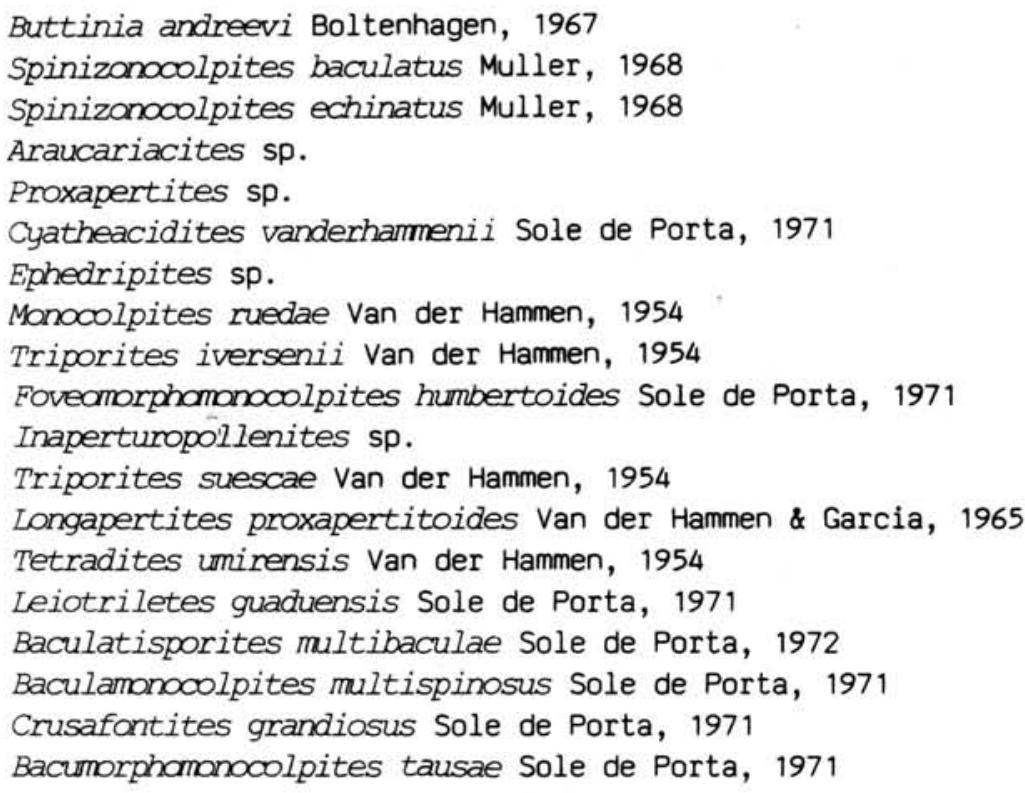

MICROPLANCKTON

Dinogymium acuminatum Evitt et al, 1967

Senegalinium bicavatum Jain \& Millepied, 1973

Achomosphaera sagena Davey \& Willians, 1966

Palaeocystodinium sp.

Ceratiopsis sp.

Andalusiella mauthei Riegel, 1974

Hystrichodinium sp.

Microforaminíferos

La asociación de Buttinia andreevi con Palaeocystodinium sp., Ceratiopsis sp. y Dinogymium acuminatum es base para asignar a éstos sedimentos una edad de Maastrichtiano Temprano. La co-occurrencia de polen y esporas con dinoflagelados y microforaminíferos sugiere que éstos sedimentos se depositaron en un ambiente marino muy cercano a la linea de costa.

FORMACION MOLINO

La Cuenca del Cesár-Ranchería es una Cuenca elongada en dirección $\mathrm{N}-65^{\circ} \mathrm{E}$, en la cual se presenta sedimentos Mesozoicos y Cenozoicos cubiertos por gruesos depósitos aluviales (Fig. 3). En total se calcula en 12.000 pies el espesor de éstos sedimentos. La Cuenca tiene un área aproximada de $9.000 \mathrm{~km}^{2}$.

En la parte Norte de la Cuenca se encuentram localizados las explotaciones carboníferas de El Cerrejón. No existe hasta el momento explotaciones petroleras en ésta Cuenca, aunque 
los pocos pozos perforados en ella han dado muestras tanto de gas como de petróleo.

La Formación Molino es una secuencia predominantemente arcillosa que alcanza 480 metros de espesor. Consiste principalmente de arcillas grises claras, grises oscuras y carbonosas con pequeñas intercalaciones de calizas y de arenas. La base de ésta secuencia arcillosa está constituída por una arena glauconítica.

En la parte inferior de la Formación Molino se han reportado foraminíferos pertenecientes a la zona de Siphogenerinoides cretacea. La parte media y superior presentan asociaciones de foraminíferos característicos de la zona de Siphogenerinoides bramlettei. Por tal motivo, se considera que la mayor parte de ésta formación es Maastrichtiana.

El tope de la Formación Molino se compone de arcillas carbonosas y calizas de aguas someras, representando con ello un cambio ambiental que va de marino batial (base de la formación) a marino somero (tope de la formación).

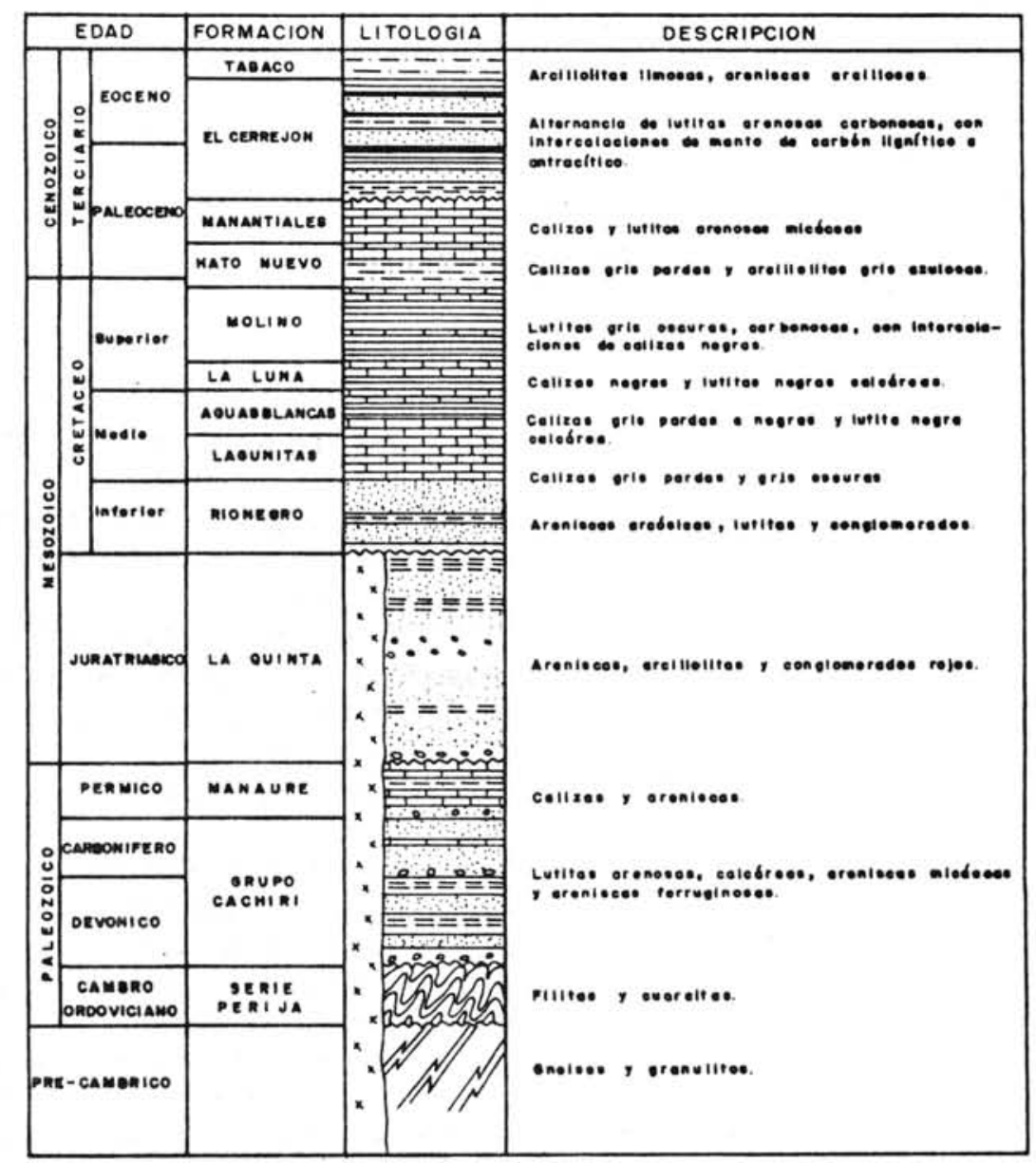

Fig. 3 - Cuenca del Cesár - columna estratigráfica generalizada.

Muestras, provenientes del tope de la Formación Molino, arrojaron buenas asociaciones palinológicas en las cuales se destaca la presencia de los siguientes palinomorfos: 
POLEN Y ESPORAS

Buttinia andreevi Boltenhagen, 1967

Spinizonocolpites baculatus Muller, 1968

Spinizonocolpites echinatus Muller, 1968

Foveotriletes margaritae Germeraad, Hopping \& Muller, 1968

Symcolporites lisamae Van der Hammen, 1954

Proteacidites dehaani Germeraad, Hopping \& Muller, 1968

Araucariacites sp.

Ephedripites sp.

Psilamonocolpites medius Van der Hammen \& Garcia, 1966

Cyatheacidites vanderhammenii Sole de Porta, 1972

Bacumorphomonocolpites tausae Sole de Porta, 1971

Echitriporites trianguliformis Van Hoeken-Klikenberg, 1964

Tetradites umirensis Van der Hammen, 1954

Leiotriletes guaduensis Sole de Porta, 1971

\section{MICROPLANCKTON}

Dinogymium acuminatum Evitt et al., 1967

Dinogymium heteriocostatum Evitt et al., 1967

Achomosphaera sagena Davey \& Willians, 1966

Senegalinium bicavatum Jain \& Millepied, 1973

Palaeocystodinium sp.

Ceratiopsis sp.

pediastrum paleogeneites Wilson \& Hoffmeister, 1953 (En REGALI et al., 1974)

La asociación recobrada de éstas muestras permite asignar a éstos sedimentos una edad Maastrichtiana. La asociación de polen, esporas, dinoflagelados y microforaminíferos sugiere que éstos sedimentos se depositaron en ambiente marino somero.

Las asociaciones palinológicas encontradas en las muestras de la Formación Molino son muy similares a las asociaciones palinológicas encontradas en la muestras de la parte superior de la Formación Colón. La única diferencia apreciable, consiste en la disminución del contenido de microplanckton en las muestras de la Formación Molino respecto al contenido reportado en las muestras de la Formación Colón, lo cual probablemente corresponda a cambios faciales en sentido Norte-Sur.

Asociaciones palinológicas similares a las encontradas en muestras de las formaciones Molino y Colón también se han reportado en el Valle Medio del Magdalena (Formación Lisama) en la Cuenca de Bogotá (Formación Guaduas) en los Llanos Orientales (Formación Guaduas o equivalentes) y en la Cuenca del Valle Superior del Magdalena, indicando con ello que la flora Maastrichtiana fue muy homogénea a lo largo del territorio colombiano.

La Plancha I es el registro fotográfico de algunos de los elementos más característicos de las asociaciones palinológicas maastrichtianas de las Formaciones Colón y Molíno. Algunos de éstos palinomorfos han sido ampliamente documentados, tal es el caso de Spinizonocolpites baculatus, Spinizonocolpites echinatus, Buttinia andreevi y Dinogymium acuminatum, los cuales son elementos comunes en las asociaciones maastrichtianas del Norte de Suramérica. Otros elementos tales como Foveomorphomonocolpites humbertoides, Bacumorphomonocolpites tausae, Baculamonocolpites multispinosus y Baculatisporites multibaculae son elementos reportados hasta el momento únicamente de sedimentos colombianos y pueden corresponder con el desarrollo de una flora maastrichtiana local. 
Bol. IG-USP, Publ.Esp., 7:173-181, 1989

\section{AGRADECIMIENTOS}

El autor desea expresar sus sinceros agradecimentos a la Academia Colombiana de Ciencias Exactas, Físicas y Naturales y al Fondo Colombiano de Investigaciones Científicas COLCIENCIAS por el decidido apoyo que permitió la presentación de éste trabajo.

REFERENCIAS BIBLIOGRAFICAS

BOL TENHAGEN, E. (1967) Spores et Pollen du Crétacé Supérieur du Gabon. Pollen et Spores, $9(2): 335-355$.

GERMERAAD, J.H.; HOPPING, C.A.; MULLER, J. (1968) Palynology of Tertiary sediments from tropical areas. Review of Paleobotany and Palynology, 6(3-4):189-348.

MILLIOUD, M.E.; WILLIANS, G.L.; LENTIN, J.K. (1975) Stratigraphic range charts selected cretaceous dinoflagellates. American Association Stratigraphy Palynology, Contributions Series, (4):65-71.

MULLER, J. (1968) Palynology of the Pedawan and Plateau Sandstone Formations (CretaceousEocene) in Sarawak, Malaysia. Micropaleontology, 14(1):1-37.

REGALI, M. da S.P.; UESUGUI, N.; SANTOS, A. da S. (1974) Palinologia dos sedimentos mesocenozóicos do Brasil (II). Boletim Técnico da Petrobrás, 17(4):263-301.

SOLE DE PORTA, N. (1971) Algunos géneros nuevos procedente de la Formación Guaduas (Maastrichtiano-Paleoceno) de Colombia. Studia geológica, 2:133-143.

SOLE DE PORTA, N. (1972) Palinología de la Formación Cimarrona (Maastrichtiano) en el Valle Medio del Magdalena, Colombia. Studia geológica, 4:103-142.

VAN DER HAMMEN, T. \& GARCIA, C. (1966) The Paleocene pollen flora of Colombia. Leidse Geologische Mededelingen, 35: 105-116.

VAN HOEKEN-KLINGERBERG, P.M.J. (1964) A palynological investigation of some Upper Cretaceous Sediments in Nigeria. Pollen et Spores, 6(1):209-231. 
Dueñas, H.J.

PLANCHA I

Fig. 1 - Spinizonocolpites baculatus.

Fig. 2 - Spinizonocolpites echinatus

Fig. 3 - Crusafontites grandiosus.

Fig. 4 - Buttinia andreevi.

Fig. 5 - Foveamorphomonocolpites humbertoides.

Fig. 6 - Bacumorphomonocolpites tausae.

Fig. 7 - Baculamonocolpites multispinosus

Fig. 8 - Monocolpites ruedae.

Fig. 9 - Baculatisporites multibaculae.

Fig. 10 - Araucariacites sp.

Fig. 11 - Dinogymium acuminatum

Fig. 12 - Ceratiopsis sp. 
Bol. IG-USP, Publ.Esp., 7:173-181, 1989

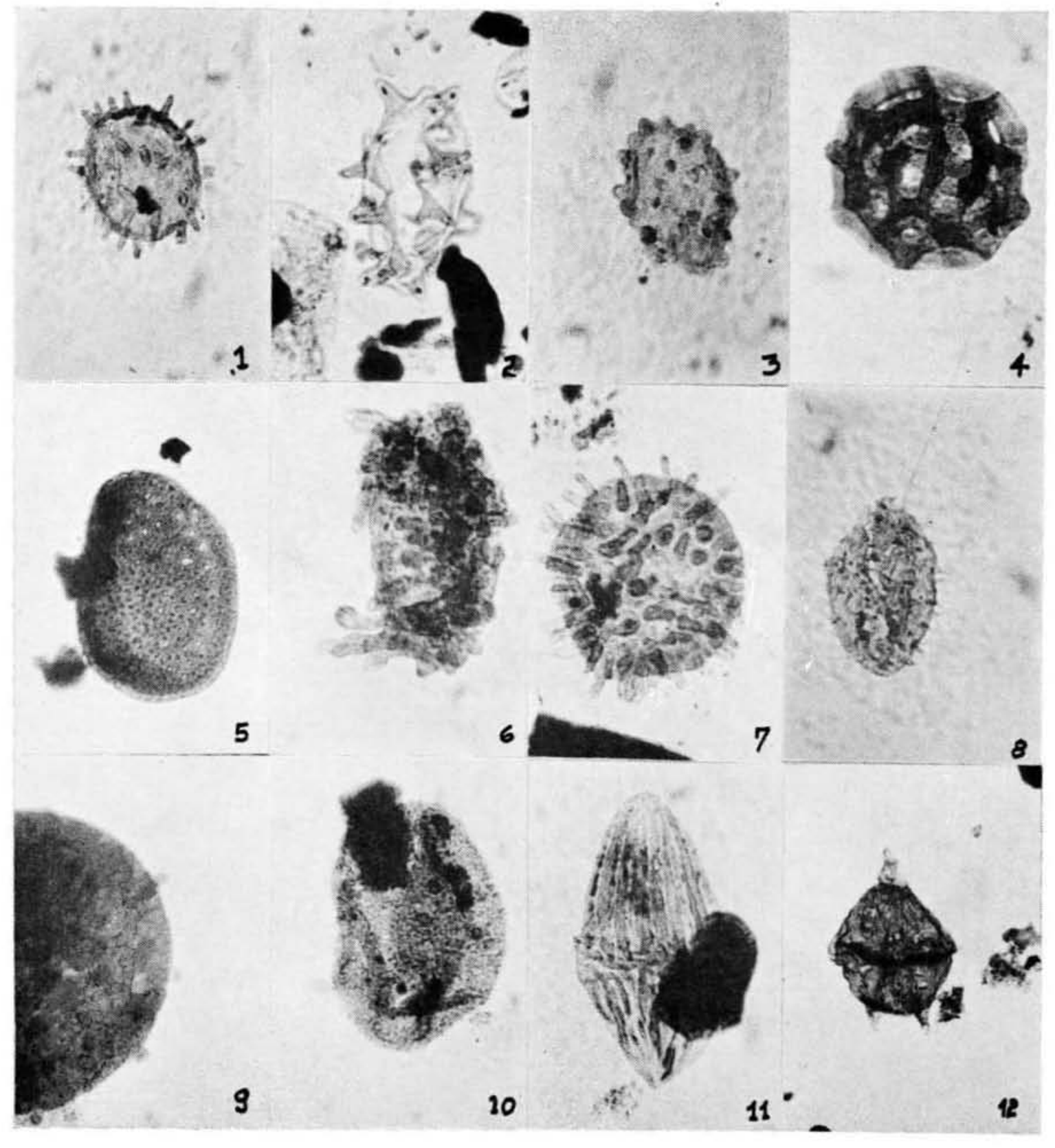

